\title{
Investigation of the Temperature Dependence of the Oscillation of the Magnetic Susceptibility in Semiconductors
}

\author{
G. Gulyamov1, U. I. Erkaboev1, N. Yu. Sharibaev'1,2 \\ ${ }^{1}$ Namangan Engineering-Pedagogical Institute, Namangan, Uzbekistan \\ ${ }^{2}$ Namangan Engineering-Technology Institute, Namangan, Uzbekistan \\ Email: Gulyamov1949@mail.ru
}

Received 1 September 2014; revised 26 September 2014; accepted 15 October 2014

Copyright (C) 2014 by authors and Scientific Research Publishing Inc.

This work is licensed under the Creative Commons Attribution International License (CC BY). http://creativecommons.org/licenses/by/4.0/

c) (i) Open Access

\section{Abstract}

The temperature dependence of the magnetic susceptibility oscillations semiconductors was considered in a quantizing magnetic field. With the help of mathematical modeling of the thermal broadening of the energy levels, the temperature dependence of the de Haas-van Alphen effect in quantizing magnetic field was investigated. The influence of temperature on the de Haas-van Alphen with the help of free energy of electrons $(\chi(H, T))$ in semiconductors was determined. Theoretical results of the mathematical simulation were compared with experimental data for bismuth. Using the proposed model of the low-temperature $(\chi(H, T))$, high-temperature oscillation magnetic susceptibility in semiconductors was calculated.

\section{Keywords}

Density of States, The Energy Spectrum, The Numerical Simulation and Experiment

\section{Introduction}

In a strong magnetic field, the energy spectrum of electrons in a semiconductor becomes quantized, so that the density of states as a function of energy acquires an oscillating character. This circumstance is the root cause of occurrence of oscillatory magnetic field dependence of a number of equilibrium and nonequilibrium quantities characterizing the state and behavior of the electrons in the crystal in a quantizing magnetic field. Currently, these oscillations are united under the general name of quantum oscillation effects [1].

Effect de Haas-van Alphen (dHvA) is a versatile and powerful tool to study the energy spectrum of the free 
electron systems in semiconductors. The first time the definition of the oscillations of the magnetic susceptibility was observed experimentally in bismuth crystals at low temperatures and strong magnetic fields [2].

dHvA effect in recent years due to intensive research of low-dimensional structures, primarily of semiconductor nanostructures in which the spectrum of the Landau levels is determined primarily positions dimensional quantization levels. [3] [4] detected dHvA oscillations at high temperatures and weak magnetic fields using measurements of the field and temperature dependences of the static magnetic susceptibility of nanoscale semiconductors.

Work [5]-[12] considered that the temperature dependence of the density of states is determined by relaxation spectroscopy of the energy levels in semiconductors. It has been shown that the density of surface states is temperature-dependent - a method of determining the density of surface states. It has been shown that due to the energy levels of thermal broadening, a discrete spectrum with a rise in temperature is transformed into a continuous energy spectrum. With the expansion of the energy spectrum of the density of states function GN has been shown that the value of the energy gap will depend on the temperature. These studies do not consider the properties of the magnetic oscillation effects in semiconductors.

The aim of this work is to study the temperature dependence of the magnetic susceptibility and the comparison of theoretical calculations with experimental results.

\section{Influence of Temperature on the Magnetic Susceptibility in Semiconductors}

Consider the free energy of the electron gas. According, the Gibbs distribution can be written as [13] [14]

$$
F=-k T \ln \sum_{n} \exp \left(-\varepsilon_{n} / k T\right)
$$

where, $\varepsilon_{n}$ - energy levels of the system.

The free energy $F$ is expressed in terms of electron density of states $N_{s}$. The free energy of an electron in a quantizing magnetic field is determined by the following formula [15]:

$$
F=n \xi+\frac{16 m^{3 / 2}(\mu * H)^{5 / 2}}{3 \pi^{2} \hbar^{3}} \int_{-\infty}^{\infty} \sum_{N}\left(\frac{\varepsilon}{2 \mu * H}-\left(N+\frac{1}{2}\right)\right)^{3 / 2} \frac{\partial f_{0}(\varepsilon)}{\partial \varepsilon} \mathrm{d} \varepsilon
$$

where, $\mu^{*}=\left(\frac{m}{m^{*}}\right) \mu_{B}$ — “effective” Bohr magneton, $\mu_{B}$-Bohr magneton, $H$-magnetic field strength, $N$ number of Landau levels,

$$
\frac{\partial f_{0}(\varepsilon)}{\partial \varepsilon}=-\frac{\exp \left(\frac{\varepsilon-\xi}{k T}\right)}{\left(\exp \left(\frac{\varepsilon-\xi}{k T}\right)+1\right)^{2} k T}
$$

Second-order derivative of $F(H, T)$ in the magnetic field strength, the magnetic susceptibility has

$$
\chi(H, T)=-\frac{\partial^{2} F(H, T)}{\partial H^{2}}
$$

The occurrence of oscillations of the magnetic susceptibility $\chi(H, T)$ can be explained qualitatively as follows.

Figure 1 shows graphs of the oscillations of the magnetic susceptibility calculated by Formulas (2)-(4) in a strong magnetic field at $T=5 \mathrm{~K}, \frac{m^{*}}{m_{0}}=1$. At such low temperatures, the influence of the thermal broadening of the weak. Figure 1 shows that with increasing magnetic field strength, the oscillation amplitude of the magnetic susceptibility $\chi(H, T)$ increases.

Figure 2 shows the oscillations of the magnetic susceptibility for different temperatures. As can be seen from these figures, at a temperature of $T=80 \mathrm{~K}$ peaks effect dHvA oscillations due to Landau quantization is do not observed.

In work [10] to determine the probability devastation energy states showed that, if $E_{0}=k \ln \left(\frac{n T_{0}}{\tau_{0}}\right)$ the 


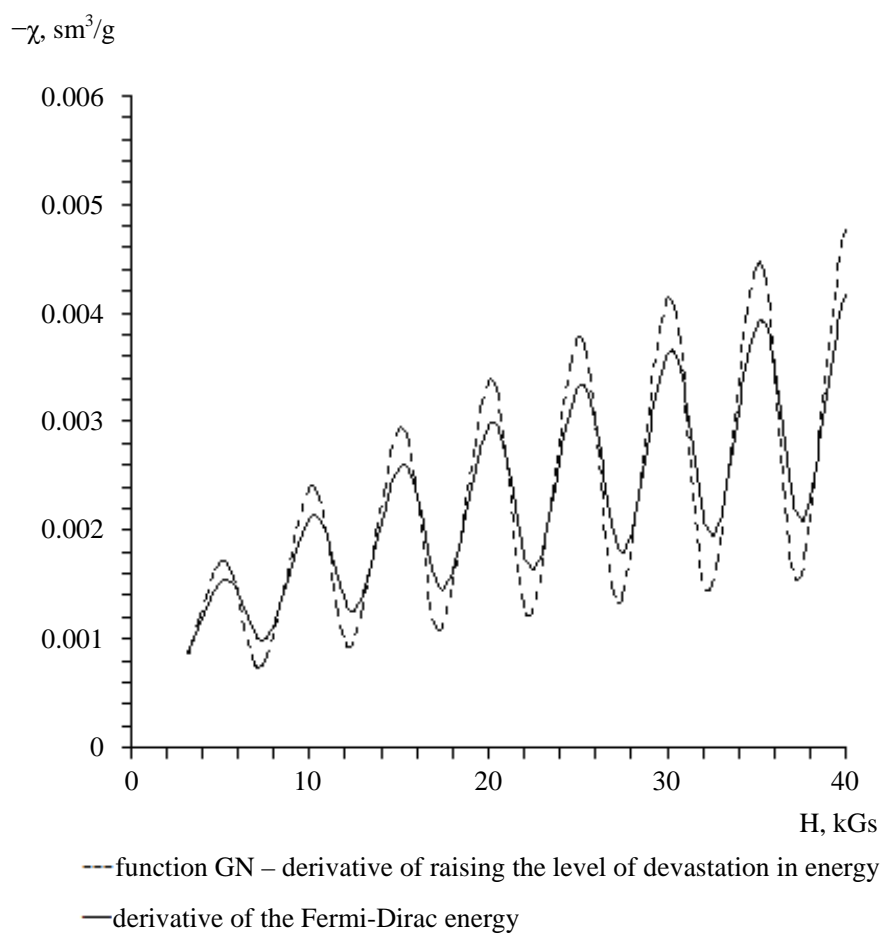

Figure 1. Dependence of the oscillation of the magnetic susceptibility of the strength magnetic field.

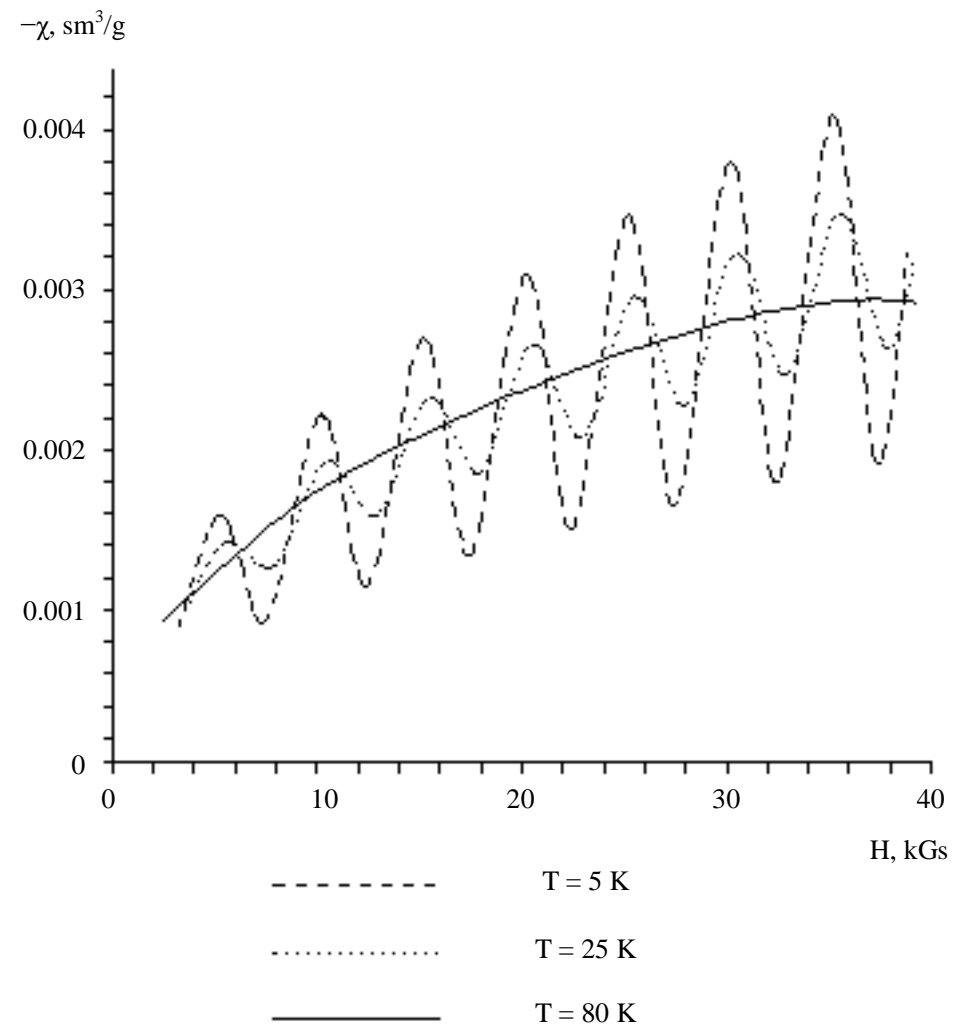

Figure 2. Change in the magnetic susceptibility at different temperatures in strong magnetic fields, calculated by Formulas (3) and (4). 
function of probability devastation energy states is $\rho\left(E_{0}(t), E, T\right)=1-\exp \left(-\exp \left(\frac{E_{0}-E}{k T}\right)\right)$ a step function, its derivative

$$
\frac{\partial \rho}{\partial E}=\mathrm{GN}\left(E_{0}(t), E, T\right)=\frac{1}{k T} \exp \left(\frac{1}{k T}\left(E_{i}-E_{0}\right)-\exp \left(\frac{1}{k T}\left(E_{i}-E_{0}\right)\right)\right)
$$

at low temperatures describes- the Dirac delta function.

Function of the form $\varphi(x)=n \exp (n x-\exp (n x))$ satisfies all the conditions necessary to obtain the deltafunction [15]:

$$
\delta(x)=\lim _{n \rightarrow \infty} n \exp (n x-\exp (n x))
$$

From here follow, the function, $\mathrm{GN}\left(T, E_{0}, E\right)$, at $T \rightarrow 0$ and $\frac{1}{k T} \rightarrow \infty$

$$
\mathrm{GN}\left(T, E_{0}, E\right)=\delta\left(E_{0}-E\right)
$$

is a delta-Dirac function. It is well known that the derivative of the energy function is the Fermi-Dirac Dirac $\delta$ function, tends to zero temperature.

Thus, to study the temperature dependence of the magnetic susceptibility of the oscillations in the Formula (2) $\frac{\partial f_{0}(E)}{\partial E}$ is replaced by the $\mathrm{GN}\left(E_{0}, E, T\right)$-function.

Then we can calculate the temperature dependence of magnetic susceptibility of the oscillation with the aid of Formulas (2), (4) and (5).

\section{Comparison of Theoretical Calculations with Experimental Results}

In the work [2] experiments were conducted on bismuth in stronger magnetic fields and low temperatures. Initial data for the application of formulas (4) and (5) is an experimental oscillation of magnetic susceptibility at a particular temperature $T$ (temperature experiment). Figure 3(a) shows the oscillation of the magnetic susceptibility at $T=14.2 \mathrm{~K}$. For analysis of the experimental results beginning requires expansion of the magnetic susceptibility in a series of $\operatorname{GN}\left(E_{0}, E, T\right)$ functions. On Figure 3(b) shows the theoretical fitting oscillation of the magnetic susceptibility obtained by the decomposition of the experimental schedule Figure 3(a) in a series of $\mathrm{GN}\left(E_{0}, E, T\right)$ functions. Comparing Figure 3(a) and Figure 3(b) shows that the oscillations of the magnetic

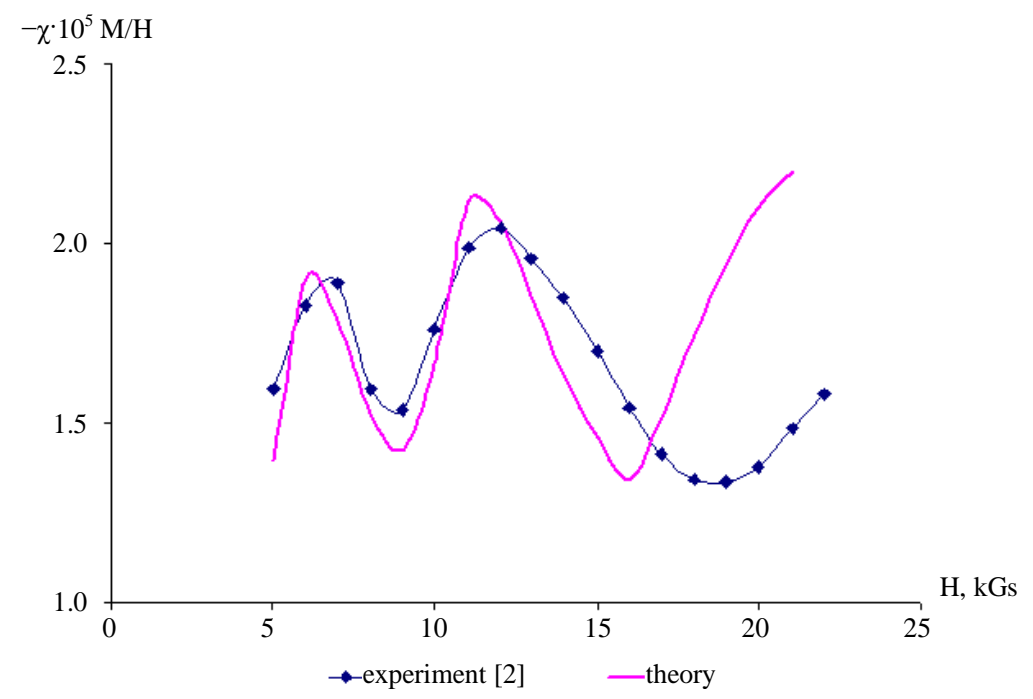

(a) 


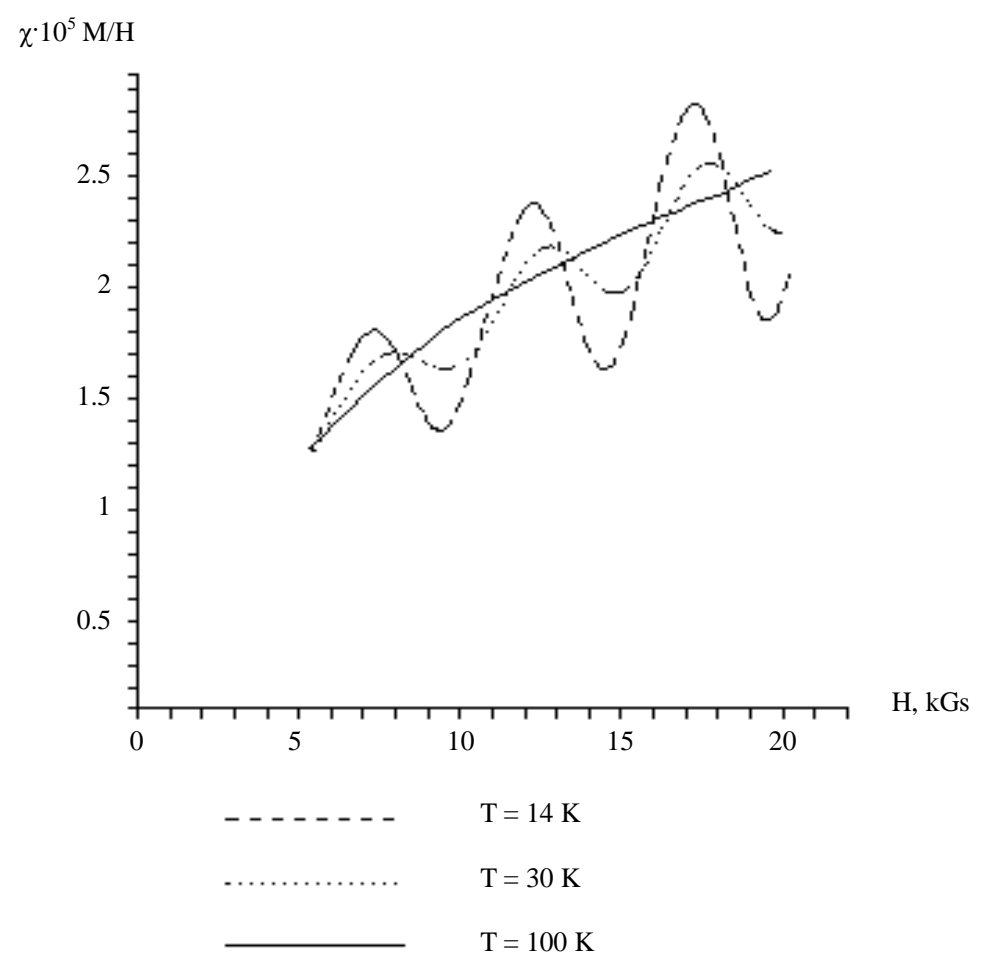

(b)

Figure 3. Changing the oscillations of magnetic susceptibility in a strong quantizing magnetic field. (a) Experiment [2] and the model calculation for bismuth at $T=14.2 \mathrm{~K}$; (b) Oscillations of magnetic susceptibility in a strong magnetic field for three different values of temperature.

susceptibility of bismuth observed at the temperature of the experiment. Because, in this experiment $\hbar \omega_{c} \approx 0,08 \mathrm{eV}$, at temperature $T=14.2 \mathrm{~K} . \frac{\hbar \omega_{c}}{k T} \approx 41, k T \ll \hbar \omega_{c}$. In this case, the Landau levels appear abruptly. With increasing temperature starts to smooth sharp spikes and at sufficiently high temperatures $k T>\hbar \omega$, $\chi(H, T)$ turns into a continuous spectrum of the magnetic susceptibility. Figure 3(b) shows $\chi(H, T)$ for different temperatures in a strong magnetic field. At a temperature of $100 \mathrm{~K}$ for bismuth oscillations of magnetic susceptibility is do not observed. Theoretical calculations for these materials are in good agreement with the experimental data.

\section{Conclusion}

The dependence of the oscillation of the magnetic susceptibility of the temperature in a quantizing magnetic field by the free energy of the electrons was investigated. It is shown that, as temperature is increased starts to smooth sharp spikes and at sufficiently high temperatures $\chi(H, T)$ turns into a continuous spectrum of oscillations of the magnetic susceptibility. Mathematical simulation of the processes with the use of experimental results for bismuth [2] at low temperatures and strong magnetic field $\chi(H, T)$, the calculated high-temperature oscillation dHvA effect. Simulation of the temperature dependence of the oscillation allowed determined magnetic susceptibility of bismuth in wide temperature ranges.

\section{References}

[1] Tsidilkovsky, I.M. (1972) Electrons and Holes in Semiconductors. Nauka, Moscow, Chapter 6, 526.

[2] Schoenberg, D. (1986) Magnetic Oscillations in Metals. Wiley, New York, Chapter 1, 25.

[3] Bagraev, N.T., Brilinskaya, E.S., Gets, D.S., Klyachkin, L.E., Malyarenko, A.M. and Novels, V.V. (2011) Fizika i 
Tekhnika Poluprovodnikov, 45, 1503-1508.

[4] Bagraev, N.T., Brilinskaya, E.S., Danilovsky, E.Yu., Klyachkin, L.E., Malyarenko, A.M. and Romanov, V.V. (2012) Fizika i Tekhnika Poluprovodnikov, 46, 90-95.

[5] Gulyamov, G. and Sharibaev, N.Yu. (2011) Fizika i Tekhnika Poluprovodnikov, 45, 178-182.

[6] Gulyamov, G., Sharibaev, N.Yu. and Erkaboev, U.I. (2012) Fyzicheckaya Injeneriya Poverkhnosti, 10, 366-370.

[7] Gulyamov, G., Karimov, I.N., Sharibaev, N.Yu. and Erkaboev, U.I. (2010) Uzbek Journal of Physics, 12, $143-146$.

[8] Gulyamov, G., Sharibaev N.Y. and Erkaboev, U.I. (2013) World Journal of Condensed Matter Physics, 3, $216-220$. http://dx.doi.org/10.4236/wjcmp.2013.34036

[9] Gulyamov, G., Erkaboev, U.I. and Sharibaev, N.Y. (2013) Physical Surface Engineering, 11, $289-292$.

[10] Gulyamov, G., Erkaboev, U.I. and Sharibaev, N.Y. (2014) Journal of Modern Physics, 5, 680-685. http://dx.doi.org/10.4236/jmp.2014.58079

[11] Gulyamov, G., Erkabaev, U.I. and Sharibaev, N.Yu. (2014) Semiconductors, 48, 1287-1292.

[12] Gulyamov, G., Sharibaev, N.Y. and Erkaboev, U.I. (2014) Fyzicheckaya Injeneriya Poverkhnosti, 11, 9-13.

[13] Landau, L.D. and Lifshitz, E.M. (1976) Statistical Physics. Nauka, Moscow, Part 1, Chapter 3, 109.

[14] Abrikosov, A.A. (1987) Fundamentals of the Theory of Metals. Nauka, Moscow, Chapter 10, 156.

[15] Anselm, A.I. (1978) Introduction to the Theory of Semiconductors. Nauka, Moscow, Chapter 6, 367. 
Scientific Research Publishing (SCIRP) is one of the largest Open Access journal publishers. It is currently publishing more than 200 open access, online, peer-reviewed journals covering a wide range of academic disciplines. SCIRP serves the worldwide academic communities and contributes to the progress and application of science with its publication.

Other selected journals from SCIRP are listed as below. Submit your manuscript to us via either submit@scirp.org or Online Submission Portal.
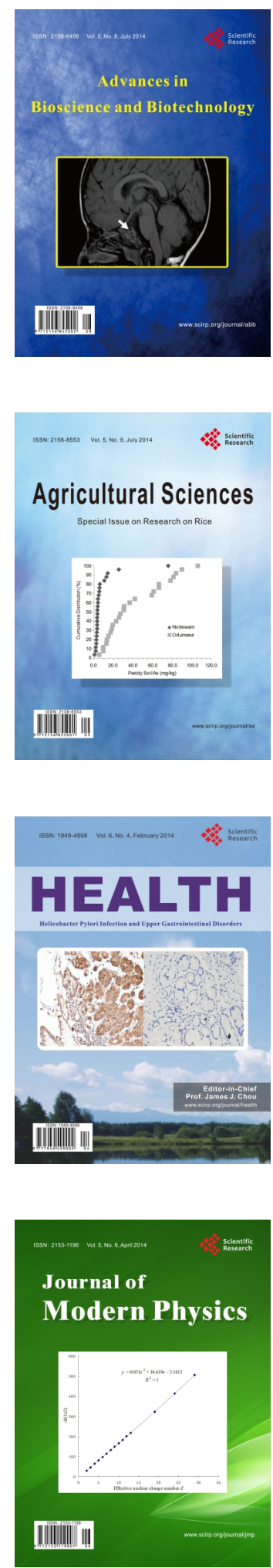
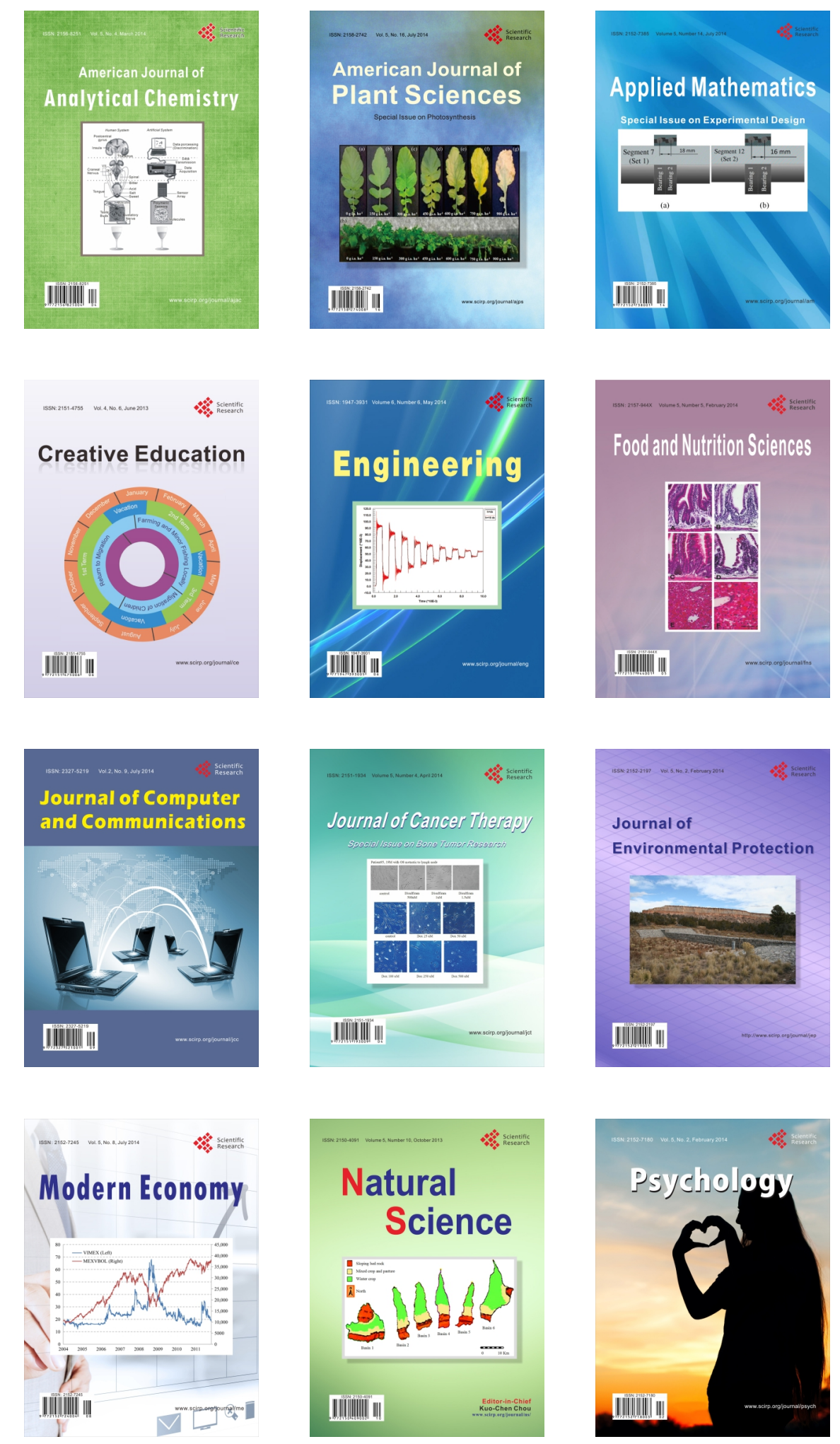